\title{
Assessment of visfatin concentrations in the serum of male psoriatic patients in relation to metabolic abnormalities
}

\author{
Katarzyna Maria Chyl-Surdacka ${ }^{A-D}$, Joanna Bartosińska ${ }^{A, C, D}$, Małgorzata Kowal ${ }^{B, C}$, \\ Joanna Przepiórka-Kosińska ${ }^{\mathrm{B}}$, Dorota Krasowska ${ }^{\mathrm{E}, \mathrm{F}}$, Grażyna Chodorowska ${ }^{\mathrm{A}, \mathrm{C}, \mathrm{E}, \mathrm{F}}$ \\ Department of Dermatology, Venerology and Paediatric Dermatology, Medical University of Lublin, Poland \\ A - research concept and design; $\mathrm{B}$ - collection and/or assembly of data; $\mathrm{C}$ - data analysis and interpretation; \\ $D$ - writing the article; $E$ - critical revision of the article; $F$ - final approval of the article
}

Address for correspondence

Katarzyna Chyl-Surdacka

kasiachyl@gmail.com

\section{Funding sources}

Medical University of Lublin, Poland, grant №. DS164.

Conflict of interest

None declared

Received on June 25, 2018

Reviewed on January 20, 2019

Accepted on August 18, 2019

Published online on February 4, 2020

Cite as

Chyl-Surdacka KM, Bartosińska J, Kowal M, PrzepiórkaKosińska J, Krasowska D, Chodorowska G. Assessment of visfatin concentrations in the serum of male psoriatic patients in relation to metabolic abnormalities. Adv Clin Exp Med. 2020:29(1):79-84. doi:10.17219/acem/111820

D0I

$10.17219 /$ acem/111820

\section{Copyright}

Copyright by Author(s)

This is an article distributed under the terms of the

Creative Commons Attribution 3.0 Unported (CC BY 3.0)

(https://creativecommons.org/licenses/by/3.0/)

\section{Abstract}

Background. Visfatin is one of the pro-inflammatory adipokines secreted by adipose tissue cells. Recent scientific research has drawn attention to the role of adipokines in the pathophysiology of metabolic disorders and their association with inflammatory diseases, including psoriasis. Visfatin may be one of the important links explaining the connection between psoriasis and diseases which are components of metabolic syndrome.

Objectives. The aim of this study was to assess the serum visfatin concentration in patients with psoriasis and to evaluate its possible correlations with parameters of metabolic syndrome and the clinical severity of psoriasis.

Material and methods. A group of 102 patients with psoriasis and a control group of 40 healthy subjects were examined. The clinical severity of psoriasis was assessed according to Psoriasis Area and Severity Index), BSA (Body Surface Area) and DLQI (Dermatology Life Quality Index) indicators, the presence and type of obesity, and hypertension. In both the study and control groups, laboratory tests (C-reactive protein (CRP), glucose concentration, total cholesterol, low-density-lipoprotein (LDL) cholesterol, high-densitylipoprotein (HDL) cholesterol, and triglycerides (TG)) were performed and serum visfatin concentrations were determined. The clinical data, results of laboratory tests and visfatin concentrations were then subjected to statistical analysis.

Results. There was a significantly higher concentration of visfatin in the psoriatic patients $(p<0.001)$ than in the control group. Significant positive correlations between visfatin concentration and PASI ( $p=0.008)$ and BSA ( $p=0.007$ ) were observed. In the psoriatic group, there were positive correlations between the concentrations of visfatin and the concentrations of CRP $(p=0.008)$ and total cholesterol $(p=0.002)$. Visfatin concentration was elevated in the psoriatic patients who had elevated total cholesterol ( $p=0.001), \mathrm{LDL}$ cholesterol $(p=0.012)$ and TG levels $(p=0.001)$ compared to the psoriatic patients with normal levels of these lipid profile components.

Conclusions. The results indicate the possible participation of visfatin in pathophysiological and inflammatory processes in the course of psoriasis. Adipokines may be an important link connecting psoriasis with coexisting metabolic disorders.

Key words: psoriasis, visfatin, metabolic syndrome 


\section{Introduction}

Psoriasis is a chronic, genetically determined inflammatory skin disease with autoimmune involvement and a pathogenesis which still has not been completely revealed. Recent literature data indicates that psoriasis can be included in the group of chronic inflammatory systemic diseases (CIDs). The presence of various mediators - including cytokines and adipokines - responsible for systemic inflammation and its metabolic consequences has been demonstrated not only in the affected skin but also in peripheral circulation. Inflammatory mediators, also secreted by adipose tissue cells, participate in inducing vascular endothelial dysfunction and insulin resistance, increasing the concentration of thrombogenic factors and promoting oxidative stress and lipid oxidation disorders. Adipokines may be an important link connecting psoriasis with coexisting metabolic disorders.

Visfatin is a $52 \mathrm{kDa}$ protein composed of 473 amino acids secreted mainly by hepatocytes, macrophages and adipocytes of visceral adipose tissue. ${ }^{1-8}$ The intracellular form of the protein is crucial in cellular metabolism regulation, adaptation to extracellular stressors and cell survival. The extracellular form detected in the circulation and extracellular environment has an influence on the inflammatory and metabolic processes. ${ }^{3,5,9,10}$ Some studies have indicated the involvement of visfatin in the pathogenesis of abdominal obesity, atherosclerosis, type 2 diabetes mellitus, and vascular and inflammatory diseases, ${ }^{1,4-6,9,11}$ because the protein has enzymatic, metabolic, inflammatory, and immunomodulatory properties. ${ }^{5}$ Moreover, the protein affects vascular endothelial cells and vascular smooth muscle cells (VSMC) and it stimulates neoangiogenesis. ${ }^{5,12}$ Visfatin contributes to increased expression of adhesion molecules, i.e., intercellular adhesion molecule 1 (ICAM-1), vascular cell adhesion molecule 1 (VCAM-1), selectin, and vascular endothelial growth factor (VEGF), which results in increased recruitment and adherence of the immune cells to the vascular endothelium, increased proliferation and migration of the endothelium, and the formation of new blood vessels. Visfatin affects the inflammatory reaction in the vessel, inducing increased secretion of the molecules interleukin 6 (IL-6), IL-8, monocyte chemoattractant protein 1 (MCP1), tumor necrosis factor $\alpha$ (TNF- $\alpha$ ), matrix metalloproteinase 2 (MMP-2), and MMP-9 by endothelial cells and monocytes, ${ }^{5,13-17}$ and activating nicotinamide adenine dinucleotide oxidase. ${ }^{3}$ Exogenous protein administration has been shown to increase the synthesis of the inducible nitric oxide synthase (iNOS) enzyme, impairing the production of nitric oxide (NO) and enhancing oxidative stress in atherosclerotic plaque. ${ }^{4,5,9,12,13,18,19}$ On the other hand, recently published scientific reports point to a beneficial effect of visfatin on the course of ischemic processes. It seems that due to its antiapoptotic properties, it promotes the prolonged survival of myocardial and neuronal cells; therefore, the protein may prove to be a useful therapeutic tool in reducing the area of myocardial or cerebral ischemia in the course of acute necrosis of these organs..$^{3,5,20-22}$ The proangiogenic, antiapoptotic and pro-inflammatory properties of visfatin may indicate possible involvement in the development of cancer. ${ }^{5,9}$

\section{Objectives}

Due to the relationship between psoriasis and metabolic syndrome, as well as the important role of visfatin in the induction of vascular endothelial dysfunction, our research was undertaken to investigate the relationship between the serum visfatin concentrations, psoriasis and individual components of metabolic syndrome.

\section{Material and methods}

The study included 102 adult, male psoriatic patients and 40 healthy, age-matched men. The patients with psoriasis were interviewed about the duration of their disease, the coexistence of psoriatic arthritis, any medications taken, and the presence of systemic comorbidities - with special attention to disorders that are components of metabolic syndrome, such as hypertension, diabetes, lipid disorders, and coexisting ischemic heart disease. The clinical condition of the patients was assessed by measuring blood pressure; determining the extent and severity of psoriatic skin lesions with the use of the indicators PASI (Psoriasis Area and Severity Index), BSA (Body Surface Area) and DLQI (Dermatology Life Quality Index); and assessing the presence and extent of obesity with body mass index (BMI) and waist-to-hip ratio (WHR). Patients with BMI values above 30 were considered obese. The WHR values higher than 1 confirmed the presence of abdominal obesity. The PASI values exceeding 10 indicated a moderate course of the disease, whereas patients with values over 18 were classified as having severe psoriasis. Patients in both the psoriasis group and the control group were assessed for serum concentrations of visfatin and laboratory parameters: C-reactive protein (CPR) concentration and components of the lipid profile (total cholesterol, high-density-lipoprotein (HDL) cholesterol, low-density-lipoprotein (LDL) cholesterol, triglycerides (TG), and glucose). Serum visfatin concentrations were determined with the immunoenzymatic enzyme-linked immunosorbent assay (ELISA) method according to the manufacturer's recommended procedure using an AdipoGen Nampt (Visfatin/ PBEF) human ELISA Kit (test sensitivity: 3.0 pg/mL, absorbance reading: $450 \mathrm{~nm}$ ) (AdipoGen Corp., San Diego, USA). For the analysis of the results obtained, using IBM SPSS software v. 19 (IBM Corp., Armonk, USA), statistical significance was assumed at a p-value $<0.05$. 


\section{Results}

There was a significantly higher concentration of visfatin in psoriatic patients than in the control group $(\mathrm{p}<0.001)$ (Fig. 1, Table 1). In the study population, a significant correlation between serum visfatin concentration and CRP value ( $\mathrm{p}=0.008$ ) was found; a positive correlation was also observed between the serum concentration of visfatin and PASI ( $\mathrm{p}=0.008$ ) (Fig. 2) and BSA ( $\mathrm{p}=0.007$ ) (Fig. 3). The protein concentrations were also significantly higher in the patients with severe psoriasis, as measured with PASI, compared to patients with mild disease severity $(\mathrm{p}=0.013)$. However, no significant correlations were observed between the serum concentration of visfatin and DLQI $(p=0.544)$

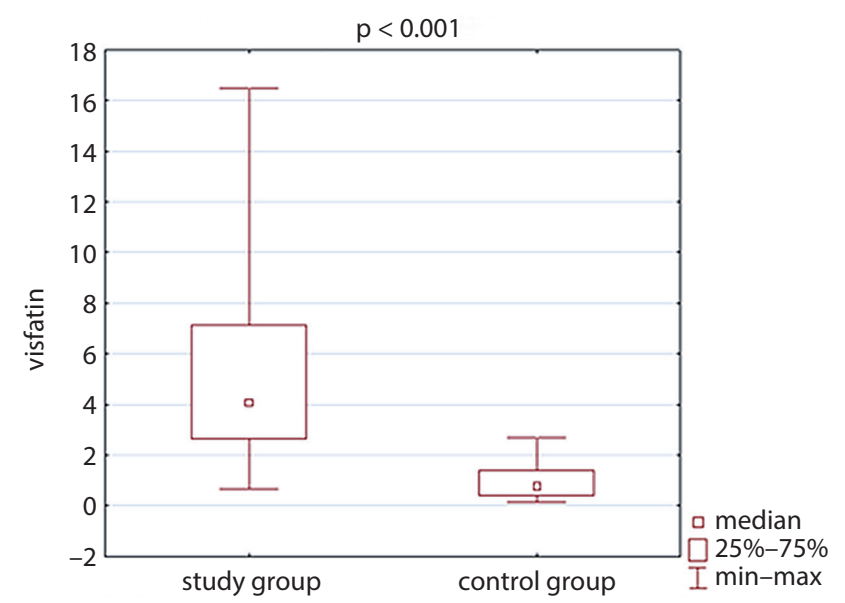

Fig. 1. Assessment of visfatin concentration in the experimental and control groups

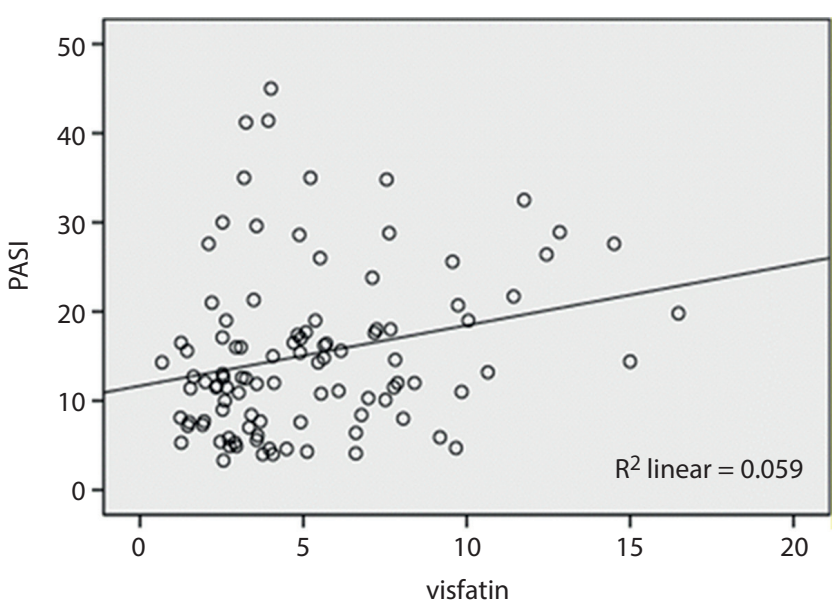

Fig. 2. Correlation between visfatin and PASI values

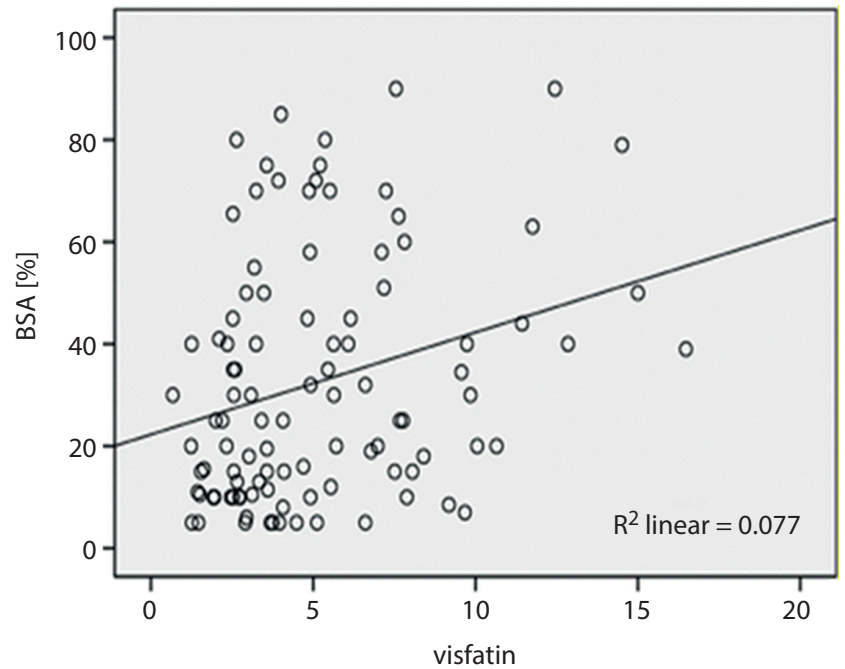

Fig. 3. Correlation between visfatin and BSA values

or duration of the disease $(\mathrm{p}=0.576)$. There were no correlations between the serum concentration of visfatin and BMI or fat distribution (WHR) ( $\mathrm{p}=0.380$ and $\mathrm{p}=0.095$, respectively). In the psoriatic group, there was a significant positive correlation between serum visfatin concentration and total cholesterol level ( $p=0.002)$. Serum visfatin concentration was significantly higher in the psoriatic patients who also had elevated total cholesterol, LDL cholesterol and TG levels compared to the patients with normal values of these parameters $(p=0.001, p=0.012$ and $p=0.001$, respectively). The concentration of visfatin was not different in the psoriatic patients with and without concomitant arterial hypertension $(\mathrm{p}=0.749)$.

\section{Discussion}

Visfatin belongs to the group of adipokines produced by adipose tissue cells. The literature indicates visfatin involvement in the pathogenesis of many inflammatory diseases, e.g., ulcerative colitis, Crohn's disease or hepatitis C. ${ }^{9,17}$ It is possible that the protein also participates in pathophysiological processes in psoriasis. In our own study, significantly higher concentrations of visfatin were observed in the group of psoriatic patients than in the control group. Moreover, in the experimental group, positive correlations between serum visfatin concentration and PASI and BSA values were also found.

Similar results have been presented by Ismail et al. ${ }^{23}$ in investigating the serum concentrations of visfatin and

Table 1. The concentration of visfatin in patients with psoriasis and in the control group

\begin{tabular}{|l|c|c|c|c|c|c|c|}
\multicolumn{1}{|c|}{ Visfatin } & $\begin{array}{c}\text { Number } \\
\text { of patients }\end{array}$ & Average & Median & Minimum & Maximum & $\begin{array}{c}\text { Lower } \\
\text { quartile }\end{array}$ \\
\hline Experimental group & 102 & 5.19 & 4.07 & 0.67 & 16.48 & 2.66 \\
\hline Control group & 40 & 0.93 & 0.78 & 0.15 & 2.67 & 0.40 \\
\hline
\end{tabular}

$Z=8.694 ; p<0.001 ; S D-$ standard deviation. 
omentine in a group of 46 patients with plaque psoriasis. The authors found a significantly elevated concentration of visfatin in the psoriatic patients compared to a group of 42 healthy people. In addition, they observed positive correlations between visfatin concentration and PASI value and disease duration. Moreover, visfatin concentration was significantly higher in the patients with severe psoriasis. The results of our own study are also in agreement with the ones presented by Okan et al. ${ }^{24}$ who observed a positive correlation between visfatin concentration and disease severity in a group of 45 patients with plaque psoriasis, as assessed with PASI.

Campanati et al. ${ }^{25}$ evaluated the serum adipokines in patients with psoriasis before and after the 24-week period of treatment with TNF- $\alpha$ inhibitors. As in our own study, elevated concentrations of visfatin were found in the patients with psoriasis compared to the control group. A significant difference in the serum visfatin concentration between the experimental and control groups was maintained even after 24 weeks of biological treatment. The authors, however, did not find any relationship between the concentration of visfatin and the severity of the disease.

On the contrary, Sereflikan et al..$^{26}$ assessed the concentrations of visfatin, adiponectin and TNF- $\alpha$ in the group of 42 psoriatic patients and noticed that although the levels of visfatin in the experimental group were higher than in the controls, the difference was not statistically significant. The authors did not observe a significant correlation between serum visfatin concentration and PASI value.

In our study, higher concentrations of CRP were recorded in the study group than in the control group $(\mathrm{p}=0.008)$, and a positive correlation between serum CRP and visfatin concentration ( $\mathrm{p}=0.008)$ was found. Similarly, Catalán et al., ${ }^{27}$ studying a group of 30 obese men and a 14 controls, found a positive correlation between visfatin concentration and not only CRP concentration but other inflammatory parameters as well, i.e., the levels of fibrinogen and von Willebrand factor. Lu et al. ${ }^{28}$ investigated a group of 173 patients with chronic kidney disease and also showed positive correlations between serum visfatin concentration and CRP value and the total number of leukocytes and neutrocytes.

Visfatin is known to be a protein produced by macrophages of the visceral adipose tissue, among other tissues. However, previous studies have yielded conflicting data on concentrations of the protein in correlation with BMI and adipose tissue distribution. In our own study, there was no statistically significant correlation between serum visfatin concentration and BMI $(\mathrm{p}=0.380)$ or WHR $(p=0.095)$ in the group of psoriatic patients. This relationship was still not observed after dividing the study population into subgroups of normal body mass, overweight and obesity, as well as subgroups with gluteal-femoral and abdominal fat distribution.

Other authors ${ }^{23,25,29}$ investigating the concentration of visfatin in patients with psoriasis and lipid disorders, insulin resistance and hypertension obtained similar results. In the psoriatic patients, the authors did not find any correlation between serum visfatin concentration and obesity rate. Similarly, Ozal et al. ${ }^{30}$ did not observe a significant correlation with BMI values in patients with hypertension with and without coexisting obesity.

Interestingly, the results obtained by Sereflican et al. ${ }^{26}$ were different from the results of the present study and the abovementioned ones. The authors observed a correlation between serum visfatin concentration and BMI value in a group of 42 Turkish psoriatic patients. Similarly, elevated concentrations of visfatin in groups of obese patients and a positive correlation of visfatin with BMI and abdominal obesity have also been observed by other authors. ${ }^{27,31-35}$

Dyslipidemia is an essential element of metabolic syndrome, occurring more often in people suffering from psoriasis. Data on the relationship between serum visfatin level and lipid profile components is divergent and does not give a definite answer as to whether the protein can serve as a predictor of these disorders.

While studying lipid disorders, we found significantly higher total cholesterol levels in our group of psoriatic patients in comparison to the control group $(\mathrm{p}<0.001)$. There was also a significant positive correlation between serum visfatin and total cholesterol concentrations ( $\mathrm{p}=0.002)$. However, no significant correlation was observed between serum visfatin concentration and the level of LDL cholesterol ( $p=0.059)$, HDL cholesterol $(\mathrm{p}=0.249)$ and TG ( $\mathrm{p}=0.224)$. However, after dividing the subjects into groups with normal and abnormal lipid profiles, a significantly higher concentration of visfatin in patients with both psoriasis and elevated total cholesterol $(p=0.001)$, LDL cholesterol $(\mathrm{p}=0.012)$ and TG $(\mathrm{p}=0.001)$ was found in comparison with the patients without dyslipidemia.

While Catalán et al. ${ }^{27}$ showed a positive correlation between the concentration of visfatin and lipid profile components - i.e., total cholesterol, HDL cholesterol and TG - in their study of 30 individuals with morbid obesity, independent of other factors, Coban et al. observed a negative correlation between visfatin concentration and total cholesterol and LDL cholesterol levels in a group of psoriatic patients. ${ }^{36}$

Some data indicate a significant effect of visfatin on the cardiovascular system. ${ }^{5}$ In addition to involvement in the induction of vascular endothelial dysfunction, vascular damage and atherosclerosis, there is more and more evidence on the association of this adipokine with the regulation of arterial blood pressure, though it remains the subject of numerous controversies.

In our own analysis, after dividing the patients into those suffering from arterial hypertension and those without the disease, there was no statistically significant difference in visfatin concentration between these subgroups $(\mathrm{p}=0.749)$. Similar results were presented by Kocelak et al. ${ }^{37}$ from a study on a large group of 2,789 older Polish 
patients. The authors divided the patients into groups composed of people without hypertension, patients with pharmacologically regulated hypertension and those untreated for the disease. The authors did not observe significantly higher visfatin values in patients with hypertension compared to patients not suffering from the disease, nor did they find any influence of treatment or disease severity on the hormone levels. In contrast, significantly higher visfatin values were observed in patients with elevated CRP levels (hs-CRP) and homeostatic model assessment of insulin resistance (HOMA-IR).

Likewise, other authors have reported that there was no significant correlation between serum visfatin concentrations and systolic and diastolic blood pressure values in groups of patients with ischemic heart disease and metabolic disorders. ${ }^{28,38,39}$

Different results were presented by Ozal et al. ${ }^{30}$ when they compared the serum visfatin concentrations of a group of 71 patients with refractory hypertension with a group of 94 pharmacologically controlled patients. The results showed significantly higher adipokine concentrations in the first group and a positive correlation with systolic and diastolic blood pressure. Moreover, in this study, visfatin turned out to be an independent predictor of the severity of hypertension.

Liakos et al., ${ }^{40}$ whose results were consistent with the previous authors' reports, compared serum visfatin and apelin concentrations in patients with upper-limit values with the concentrations in healthy subjects. Significantly higher concentrations of visfatin and lower apelin concentrations were found in the study group, suggesting a possible contribution of both adipokines in the prognosis of hypertension.

\section{Conclusions}

Visfatin is a pro-inflammatory adipokine and some literature data indicate its involvement in metabolic disorders, autoimmune diseases and cancer. In our study, the significantly higher concentrations of visfatin found in the patients with psoriasis compared to the control group indicate a possible involvement of this adipokine in the pathophysiological processes present in the course of psoriasis. Since positive correlations between serum visfatin concentration and CRP level and PASI and BSA values have been demonstrated, visfatin may prove to be a useful biomarker of systemic inflammation and psoriasis severity.

\section{References}

1. Al-Suhaimi EA, Shehzad A. Leptin, resistin and visfatin: The missing link between endocrine metabolic disorders and immunity. Eur JMed Res. 2013;18:12-25.

2. Berndt J, Klöting N, Kralisch S, et al. Plasma visfatin concentrations and fat depot-specific mRNA expression in humans. Diabetes. 2005; 54(10):2911-2916.
3. Carbone F, Liberale L, Bonaventura A, et al. Regulation and function of extracellular nicotinamide phosphoribosyltransferase/visfatin. Compr Physiol. 2017;7(2):603-621.

4. Hognogi LD, Simiti LV. The cardiovascular impact of visfatin - an inflammation predictor biomarker in metabolic syndrome. Clujul Med. 2016;89(3):322-326.

5. Romacho T, Sánchez-Ferrer CF, Peiró C. Visfatin/Nampt: An adipokine with cardiovascular impact. Mediators Inflamm. 2013;2013:946427.

6. Saboori S, Hosseinzadeh-Attar MJ, Yousefi Rad E, Hosseini M, Mirzaei K, Ahmadivand $Z$. The comparison of serum vaspin and visfatin concentrations in obese and normal weight women. Diabetes Metab Syndr. 2015;9(4):320-323.

7. Tan BK, Chen J, Digby JE, Keay SD, Kennedy CR, Randeva HS. Increased visfatin messenger ribonucleic acid and protein levels in adipose tissue and adipocytes in women with polycystic ovary syndrome: Parallel increase in plasma visfatin. J Clin Endocrinol Metab. 2006;91(12): 5022-5028.

8. Ziora K, Oświęcimska J, Świętochowska E, et al. Stężenie wisfatyny w surowicy krwi u dziewcząt z otyłością prostą. Endokrynol Pediatr. 2011;10(2):17-25.

9. Bułdak RJ, Polaniak R, Kukla M, Żwirska-Korczala K. Wisfatyna: enzym, cytokina czy adipokina? Funkcje biologiczne wisfatyny in vitro. www. endokrynologia.viamedica.pl 2011.

10. Revollo JR, Grimm AA, Imai S. The NAD biosynthesis pathway mediated by nicotinamide phosphoribosyltransferase regulates Sir2 activity in mammalian cells. J Biol Chem. 2004;279(49):50754-50763.

11. Uslu S, Kebapçi N, Kara M, Bal C. Relationship between adipocytokines and cardiovascular risk factors in patients with type 2 diabetes mellitus. Exp TherMed. 2012;4(1):113-120.

12. Kim SR, Bae SK, Choi KS, et al. Visfatin promotes angiogenesis by activation of extracellular signal-regulated kinase 1/2. Biochem Biophys Res Commun. 2007;357(1):150-156.

13. Adya R, Tan BK, Punn A, Chen J, Randeva HS. Visfatin induces human endothelial VEGF and MMP-2/9 production via MAPK and PI3K/Akt signalling pathways: Novel insights into visfatin-induced angiogenesis. Cardiovasc Res. 2008;78(2):356-365.

14. Galkina E, Ley K. Vascular adhesion molecules in atherosclerosis. Arterioscler Thromb Vasc Biol. 2007;27(11):2292-2301.

15. Lee WJ, Wu CS, Lin H, et al. Visfatin-induced expression of inflammatory mediators in human endothelial cells through the NF-kappaB pathway. Int J Obes (Lond). 2009;33(4):465-472.

16. Liu SW, Qiao SB, Yuan JS, Liu DQ. Visfatin stimulates production of monocyte chemotactic protein-1 and interleukin- 6 in human vein umbilical endothelial cells. Horm Metab Res. 2009;41(4):281-286.

17. Moschen AR, Kaser A, Enrich B, et al. Visfatin, an adipocytokine with proinflammatory and immunomodulating properties. J Immunol. 2007;178(3):1748-1758.

18. Romacho $T$, Azcutia V, Vázquez-Bella M, et al. Extracellular PBEF/ NAMPT/visfatin activates pro-inflammatory signalling in human vascular smooth muscle cells through nicotinamide phosphoribosyltransferase activity. Diabetologia. 2009;52(11):2455-2463.

19. Wang P, Xu TY, Guan YF, Su DF, Fan GR, Miao CY. Perivascular adipose tissue-derived visfatin is a vascular smooth muscle cell growth factor: Role of nicotinamide mononucleotide. Cardiovasc Res. 2009; 81(2):370-380.

20. Hausenloy DJ. Drug discovery possibilities from visfatin cardioprotection? Curr Opin Pharmacol. 2009;9(2):202-207.

21. Hsu CP, Oka S, Shao D, Hariharan N, Sadoshima J. Nicotinamide phosphoribosyltransferase regulates cell survival through NAD+ synthesis in cardiac myocytes. Circ Res. 2009;105(5):481-491.

22. Wang P, Xu TY, Guan YF, et al. Nicotinamide phosphoribosyltransferase protects against ischemic stroke through SIRT1-dependent adenosine monophosphate-activated kinase pathway. Ann Neurol. 2011;69(2):360-374.

23. Ismail SA, Mohamed SA. Serum levels of visfatin and omentin-1 in patients with psoriasis and their relation to disease severity. $\mathrm{Br} J \mathrm{Der}$ matol. 2012;167(2):436-439.

24. Okan G, Baki AM, Yorulmaz E, Doğru-Abbasoğlu S, Vural P. Serum visfatin, fetuin-A, and pentraxin 3 levels in patients with psoriasis and their relation to disease severity. J Clin Lab Anal. 2016;30(4): 284-289. 
25. Campanati A, Ganzetti G, Giuliodori K, et al. Serum levels of adipocytokines in psoriasis patients receiving tumor necrosis factor-a inhibitors: Results of a retrospective analysis. Int J Dermatol. 2015;54(7):839_ 845 .

26. Sereflican B, Goksugur N, Bugdayci G, Polat M, Haydar Parlak A. Serum visfatin, adiponectin, and tumor necrosis factor alpha (TNF-a) levels in patients with psoriasis and their correlation with disease severity. Acta Dermatovenerol Croat. 2016;24(1):13-19.

27. Catalán V, Gómez-Ambrosi J, Rodríguez A, et al. Association of increased visfatin/PBEF/NAMPT circulating concentrations and gene expression levels in peripheral blood cells with lipid metabolism and fatty liver in human morbid obesity. Nutr Metab Cardiovasc Dis. 2011;21(4):245-253.

28. Lu YC, Hsu CC, Yu TH, et al. Association between visfatin levels and coronary artery disease in patients with chronic kidney disease. Iran J Kidney Dis. 2013;7(6):446-452.

29. Coban M, Tasli L, Turgut S, Özkan S, Tunç Ata M, Akın F. Association of adipokines, insulin resistance, hypertension and dyslipidemia in patients with psoriasis vulgaris. Ann Dermatol. 2016;28(1):74-79.

30. Ozal E, Sahin I, Bolat I, et al. Visfatin levels are increased in patients with resistant hypertension and are correlated with left ventricular hypertrophy. Blood Press Monit. 2017;22(3):137-142.

31. Krzystek-Korpacka M,Patryn E, Bednarz-Misa I,HotowyK, Noczynska A. Visfatin in juvenile obesity: The effect of obesity intervention and sex. Eur J Clin Invest. 2011;41(12):1284-1291.

32. Li RZ, Ma Xn, Hu XF, et al. Elevated visfatin levels in obese children are related to proinflammatory factors. J Pediatr Endocrinol Metab. 2013;26(1-2):111-118.
33. Reda R, Shehab A, Soliman D, Gabr A, Abbass A. Serum visfatin levels in a group of Egyptian obese individuals. Egypt J Immunol. 2011;18(1): 25-32.

34. Salama HM, Galal A, Motawie AA, et al. Adipokines vaspin and visfatin in obese children. Maced J Med Sci. 2015;3(4):563-566.

35. Taşkesen D, Kirel B, Us T. Serum visfatin levels, adiposity and glucose metabolism in obese adolescents. J Clin Res Pediatr Endocrinol. 2012; 4(2):76-81.

36. Coban M, Tasli L, Turgut S, Özkan S, Tunç Ata M, Akın F. Association of adipokines, insulin resistance, hypertension and dyslipidemia in patients with psoriasis vulgaris. Ann Dermatol. 2016;28(1):74-79.

37. Kocelak P, Olszanecka-Glinianowicz M, Owczarek $A$, et al. Plasma visfatin/nicotinamide phosphoribosyltransferase levels in hypertensive elderly: Results from the PolSenior substudy. J Am Soc Hypertens. 2015;9(1):1-8.

38. Chen CC, Li TC, Li Cl, et al. The relationship between visfatin levels and anthropometric and metabolic parameters: Association with cholesterol levels in women. Metabolism. 2007;56(9):1216-1220.

39. Fadaei R, Parvaz E, Emamgholipour $S$, et al. The mRNA expression and circulating levels of visfatin and their correlation with coronary artery disease severity and 25-hydroxyvitamin D. Horm Metab Res. 2016;48(4):269-274.

40. Liakos $\mathrm{Cl}$, Sanidas EA, Perrea DN, et al. Apelin and visfatin plasma levels in healthy individuals with high normal blood pressure. Am J Hypertens. 2016;29(5):549-552. 